\title{
The Third Interplanetary Network
}

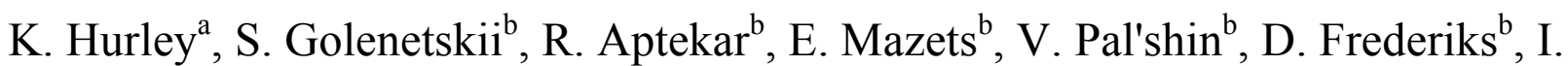

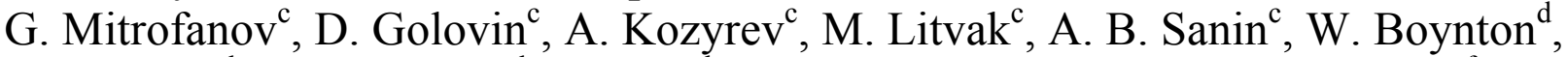

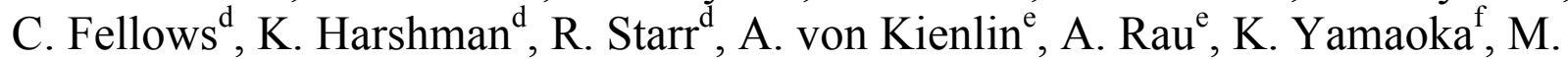
Ohno $^{\mathrm{g}}$, Y. Fukazawa ${ }^{\mathrm{h}}$, T. Takahashi ${ }^{\mathrm{g}}$, M. Tashiro ${ }^{\mathrm{i}}$, Y. Terada ${ }^{\mathrm{i}}$, T. Murakami ${ }^{\mathrm{j}}, \mathrm{K}$. Makishimak $^{\mathrm{k}}$, S. Barthelmy ${ }^{1}$, J. Cummings ${ }^{1}$, N. Gehrels ${ }^{1}$, H. Krimm ${ }^{1}$, T. Cline ${ }^{1, m}$, J. Goldsten $^{\mathrm{n}}$, E. Del Monte ${ }^{\mathrm{o}}$, M. Feroci ${ }^{\mathrm{o}}$, M. Marisaldi ${ }^{\mathrm{p}}$, M. Briggs ${ }^{\mathrm{q}}, \mathrm{V}$.

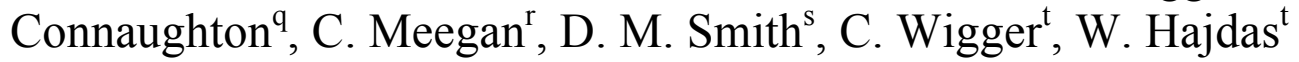

${ }^{a}$ UC Berkeley Space Sciences Laboratory, Berkeley, CA 94720-7450, U.S.A.

${ }^{b}$ Ioffe Physico-Technical Institute of the Russian Academy of Sciences, St. Petersburg, 194021, Russian Federation

${ }^{c}$ Space Research Institute, 84/32, Profsoyuznaya, Moscow 117997, Russian Federation

${ }^{d}$ University of Arizona, Department of Planetary Sciences, Tucson, AZ 85721, U.S.A.

${ }^{e}$ Max-Planck-Institut für extraterrestrische Physik, Giessenbachstrasse, Postfach 1312, Garching, 85748 Germany

${ }^{f}$ Department of Physics and Mathematics, Aoyama Gakuin University, 5-10-1 Fuchinobe, Japan

${ }^{g}$ ISAS/JAXA, 3-1-1 Yoshinodai, Sagamihara, Kanagawa 229-8510, Japan

${ }^{h}$ Dept. of Physics, Hiroshima University, 1-3-1 Kagamiyama, Higashi-Hiroshima, Hiroshima 739-8526, Japan

${ }^{i}$ Dept. of Physics, Saitama University, 255 Shimo-Okubo, Sakura-ku, Saitama-shi, Saitama 338-8570, Japan

${ }^{j}$ Dept. of Physics, Kanazawa University, Kadoma-cho, Kanazawa, Ishikawa 920-1192, Japan

${ }^{k}$ Makishima Cosmic Radiation Laboratory, RIKEN, 2-1 Hirosawa, Wako, Saitama 351-0198, Japan

${ }^{l}$ NASA Goddard Space Flight Center, Code 661, Greenbelt, MD 20771, U.S.A.

${ }^{m}$ Emeritus

${ }^{n}$ Applied Physics Laboratory, Johns Hopkins University, Laurel, MD 20723, U.S.A.

${ }^{\circ} I A S F / I N A F$, Roma, I-00133, Italy

${ }^{p}$ IASF/INAF, 40129 Bologna, Italy

${ }^{q}$ University of Alabama in Huntsville CSPAR, Huntsville, AL, U.S.A.

${ }^{r}$ Universities Space Research Association, Huntsville, AL, U.S.A.

${ }^{s}$ Physics Dept. \& Santa Cruz Institute for Particle Physics, UC Santa Cruz, Santa Cruz, CA 95064, U.S.A

${ }^{t}$ Paul Scherrer Institute, 5232 Villigen PSI, Switzerland

\begin{abstract}
The 3rd interplanetary network (IPN), which has been in operation since 1990, presently consists of 9 spacecraft: AGILE, Fermi, RHESSI, Suzaku, and Swift, in low Earth orbit; INTEGRAL,in eccentric Earth orbit with apogee 0.5 light-seconds; Wind, up to $\sim 7$ light-seconds from Earth; MESSENGER, en route to Mercury; and Mars Odyssey, in orbit around Mars. The IPN operates as a full-time, all-sky monitor for transients down to a threshold of about $6 \times 10^{-7} \mathrm{erg} \mathrm{cm}^{-2}$ or 1 photon $\mathrm{cm}^{-2} \mathrm{~s}^{-1}$. It detects $\sim 335$ cosmic gamma-ray bursts per year. These events are generally not the same ones detected by narrower field of view instruments such as Swift, INTEGRAL IBIS, SuperAGILE, and MAXI; the localization accuracy is in the several arcminute and above range. The data are publicly available and can be utilized for a wide variety of studies.
\end{abstract}

Keywords: gamma-rays: bursts; instrumentation.

PACS: $95.55 . \mathrm{Ka}$, 95.85.Pw 


\section{INTRODUCTION}

The 3rd Interplanetary Network (IPN) came into existence in 1990, with the launch of the Ulysses spacecraft. Its purpose is to derive the positions of fast gamma-ray transients of all kinds by triangulation. Numerous spacecraft and instruments have participated in the network since its inception: BATSE, PVO, Ginga, WATCH, SIGMA, PHEBUS, EURECA, Mars Observer, BeppoSAX, HETE, NEAR, and SROSS, to name a few. Today, the network consists of AGILE, Fermi, RHESSI, Suzaku, and Swift, in low Earth orbit; INTEGRAL, in eccentric Earth orbit with an apogee of 0.5 light seconds; Wind, up to 7 light seconds from Earth; MESSENGER, about to go into orbit around Mercury in March 2011, at distances up to almost 700 light seconds from Earth; and Mars Odyssey, in orbit around Mars, at distances up to about 1250 light seconds from Earth. Due to the large number of spacecraft, the roughly isotropic responses of the instruments aboard them, and the fact that three of them (INTEGRAL, MESSENGER, and Wind) view the entire sky without occultation by a planet, the IPN is an all-sky, full-time monitor of fast gamma-ray transient activity. Its limiting accuracy for localization is about 1', although only a few events can be localized this well, and its event detection rate is $\sim 335 /$ year, considering only those bursts detected by two or more detectors (i.e. confirmed GRBs). This makes it possible to study a wide variety of events which imaging GRB instruments like the Swift BAT, INTEGRAL-IBIS, AGILE, and MAXI, will seldom detect in their fields of view. These include very intense bursts, short bursts (the IPN detection rate of short bursts is much greater than those of imaging instruments), very long bursts, repeating sources (gravitationally lensed GRBs and bursting pulsars like GROJ1744-28 are two examples), soft gamma repeater activity, and possibly other as-yet undiscovered phenomena.

\section{SENSITIVITY AND RATES}

The IPN sensitivity to GRBs is a function of burst duration, energy spectrum, peak flux, and fluence. It can be defined in various ways, since the experiments comprising it vary widely in their properties. A convenient measure is to consider the fluences and peak fluxes of the GRBs detected by two or more IPN detectors, regardless of duration and spectrum. The efficiencies as a function of fluence and peak flux are shown in figure 1. The thresholds are $\sim 6 \times 10^{-7} \mathrm{erg} \mathrm{cm}^{-2}$ and 1 photon $\mathrm{cm}^{-2} \mathrm{~s}^{-1}$ for $50 \%$ efficiency. The IPN is sensitive to bursts whose energy spectra have $\mathrm{E}_{\text {peak }}$ above about $20 \mathrm{keV}$, and durations above 10's of milliseconds roughly, with efficiency dropping below these values due to detector design. The 3rd IPN has detected about 5250 confirmed cosmic gamma-ray bursts to date, not counting BATSE-only, Swift-only, or Fermi-only events. The Venn diagram of figure 2 illustrates the relation between Swift, Fermi, and IPN events. However, as experiments come into and leave the network, the burst detection rate changes slowly with time. This is illustrated in figure 3 , where the impact of the arrival and departure of missions like Wind (1995 -present), GRO (1991 - 2000), and BeppoSAX (1996 - 2002) can be seen in the rates.

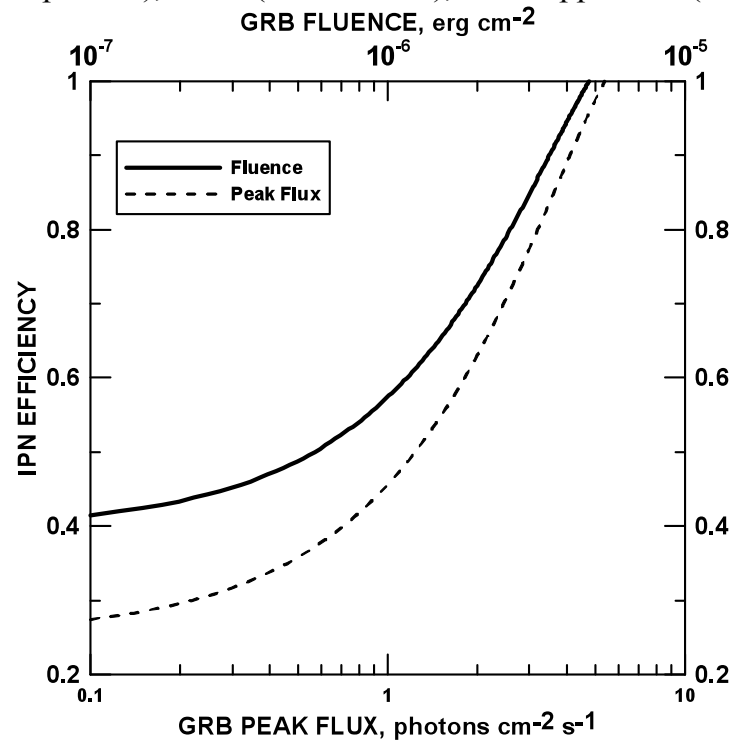

FIGURE 1. The IPN efficiency, defined as the fraction of bursts detected by two or more instruments, as a function of the GRB peak flux and fluence in the $\sim 25-150 \mathrm{keV}$ energy range, for bursts with durations in the $\sim 20 \mathrm{~ms}$ and above range. 


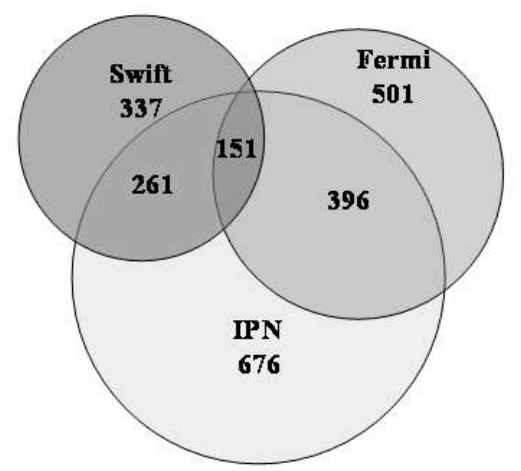

FIGURE 2. Venn diagram (not to scale) showing the numbers of Swift, IPN, and Fermi bursts between June 2008 and June 2010 and their relation. The Swift bursts are those both inside and outside of the coded FoV.

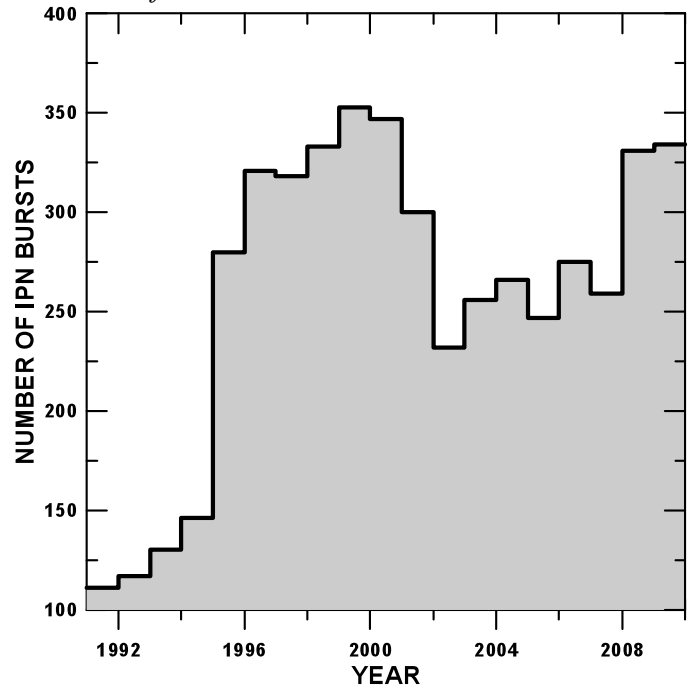

FIGURE 3. The yearly rates of bursts detected by the IPN from 1991 to 2009 . Only confirmed cosmic gamma-ray bursts have been counted (i.e., no SGRs or doubtful events). Bursts observed only by BATSE, Swift, or Fermi have not been counted.

\section{THE IPN DATA AND ITS USES}

The IPN data are public. The main components of the database are a burst list, with dates, times, and responses of the IPN spacecraft to over 8900 events, and burst localization data for 6400 bursts (these numbers do not include SGR bursts), both over the past 20 years. The former can be retrieved either at the IPN website (ssl.berkeley.edu/ipn3) or through the HEASARC "browse" interface (go to Gamma Ray Bursts from the Interplanetary Network). The latter are presently available only at the IPN website. Localization data are being added on a daily basis, but the emphasis is on completing the data for the earlier events. Some of the data have been published in a series of catalogs (10 to date, and more in preparation). For information on bursts which are not yet on the website, contact khurley@ssl.berkeley.edu. The localization data for each burst in the table contain not only IPN annuli, but all the information available; this includes BATSE and BeppoSAX error circles, Earth-blocking, and all other relevant constraints on the GRB position. Often the constraints are quite coarse, with the result that about 150 GRBs can be expected to have localizations which are consistent with any given point in the sky, when the full 20 years of data are searched. Shorter time windows, of course, have fewer bursts in them, but it is likely that at least one burst will have a localization which is consistent with any given position by chance, if a one month time window is searched. One of the current uses of IPN data is to refine Fermi GBM localizations, and assist the Fermi team in defining its systematic errors (Briggs et al. 2011). There are IPN responses to about 200 GBM bursts per year, and they are for the more intense events, where systematic uncertainties usually dominate statistical ones. Another is to search for gravitational wave signals in conjunction with GRBs, using LIGO and Virgo data (Leonor et al. 2011). About 375 bursts occurred during LIGO's $5^{\text {th }}$ Science Run, when both LIGO and Virgo were operating, and IPN data will similarly be used for LIGO's $6^{\text {th }}$ Science Run. These searches are expected to have the best 
sensitivity to gravitational radiation prior to Advanced LIGO, and the GRB sample is by far the largest used to date. The IPN data have also been used by the Milagro and ARGO YBJ groups to search for $100 \mathrm{GeV}-100 \mathrm{TeV}$ emission in conjunction with GRBs, and by the AMANDA group, to search for neutrinos (Achterberg et al. 2008, Abdo et al. 2007, Abbasi et al. 2010, Aielli et al. 2010). Because the average redshift of the current IPN bursts is $\sim 1.8$, the sample includes relatively nearby bursts, which should be an advantage for these investigations. Yet another use is to search for bursts which occurred in conjunction with Type Ib/c supernovae (Hurley \& Pian 2007, Soderberg et al. 2010, Corsi et al. 2011), or for evidence of prior activity of a newly discovered magnetar. Here the isotropic response and $\sim 100 \%$ duty cycle of the IPN are advantages, but the often poorly constrained SN explosion dates, the long time windows for the magnetar searches, and coarsely localized IPN bursts, conspire to produce numerous random coincidences which must be examined and evaluated. Finally, one of the less exciting, but still important tasks of the IPN is the non-confirmation of a GRB origin for a particular phenomenon, such as an optical or radio transient (e.g. Lorimer et al. 2007). Often, the times and positions of these phenomena are accurately known, so a search through the IPN database is unlikely to result in a random coincidence, and an unambiguous non-detection can be clearly demonstrated. FORTRAN programs are available to perform searches such as these and help with other projects. Feel free to contact khurley@ssl.berkeley.edu for more information.

\section{ACKNOWLEDGMENTS}

$\mathrm{KH}$ is grateful for IPN support from the following NASA grants and contracts: NNX09AV61G AND NNX10AR12G (Suzaku); NNX09AU03G and NNX10AU34G (Fermi); NNX10AI23G (Swift); and NNX07AR71G (MESSENGER). The Konus-Wind experiment is supported by a Russian Federal Space Agency contract and RFBR grant 09-02-00166a.

\section{REFERENCES}

1. M. Briggs et al., in preparation (2011).

2. I. Leonor et al., in preparation (2011).

3. A. Achterberg et al., Ap. J. 674, 357-370 (2008).

4. A. Abdo et al., Ap. J. 666, 361-367 (2007).

5. R. Abbasi et al., Ap. J. 710, 346-359 (2010).

6. G. Aielli et al., Astropart. Phys. 32, 47-52 (2010).

7. K. Hurley and E. Pian, "Using the Interplanetary Network to Search for SN Ic/GRB Coincidences" in Supernova 1987A: 20 Years After, edited by S. Immler, K. Weiler, and R. McCray, AIP Conference Proceedings 937, American Institute of Physics, Melvile, NY, 2007, pp. 488-491

8. A. Soderberg et al., Nature 463, 513-515 (2010).

9. A. Corsi, Ap. J., submitted (2011).

10. D. Lorimer, M. Bailes, M. McLaughlin, D. Narkevic, and F. Crawford, Science 318, 777-780 (2007). 